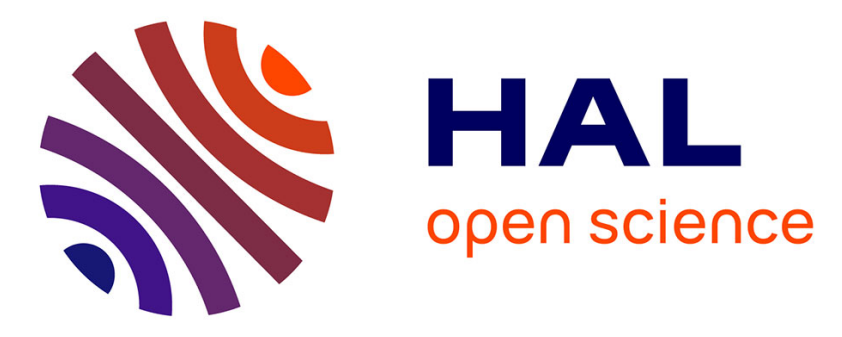

\title{
Influence of the CTAB surfactant layer on optical properties of single metallic nanospheres
}

Artur Movsesyan, Sylvie Marguet, Alina Muravitskaya, Jeremie Béal, Anne-Laure Baudrion, Pierre-Michel Adam

\section{- To cite this version:}

Artur Movsesyan, Sylvie Marguet, Alina Muravitskaya, Jeremie Béal, Anne-Laure Baudrion, et al.. Influence of the CTAB surfactant layer on optical properties of single metallic nanospheres. Journal of the Optical Society of America. A Optics, Image Science, and Vision, 2019, 36 (11), pp.C78. 10.1364/JOSAA.36.000C78 . hal-02347242

HAL Id: hal-02347242

https://hal-utt.archives-ouvertes.fr/hal-02347242

Submitted on 5 Nov 2019

HAL is a multi-disciplinary open access archive for the deposit and dissemination of scientific research documents, whether they are published or not. The documents may come from teaching and research institutions in France or abroad, or from public or private research centers.
L'archive ouverte pluridisciplinaire HAL, est destinée au dépôt et à la diffusion de documents scientifiques de niveau recherche, publiés ou non, émanant des établissements d'enseignement et de recherche français ou étrangers, des laboratoires publics ou privés. 


\title{
Influence of the CTAB surfactant layer on optical properties of single metallic nanospheres
}

\author{
Artur Movsesyan,${ }^{1,}{ }^{*}$ Sylvie Marguet, ${ }^{2}$ Alina Muravitskaya, ${ }^{3}$ \\ Jérémie Béal, ${ }^{1}$ Anne-Laure Baudrion, ${ }^{1}$ And Pierre-Michel \\ ADAM $^{1}$ \\ ${ }^{1}$ Light, Nanomaterials and Nanotechnologies (L2n), Charles Delaunay Institute, CNRS FRE 2019, \\ UniversiteİA de Technologie de Troyes, 10004 Troyes, France \\ ${ }^{2}$ 2NIMBE, CEA, CNRS, UMR 3685, UniversitÃl' Paris-Saclay, CEA Saclay, 91191 Gif-sur-Yvette cedex, \\ France \\ ${ }^{3} 3 B$. I. Stepanov Institute of Physics, National Academy of Sciences of Belarus, Nezavisimosti 68-2, Minsk \\ 220072, Belarus \\ *artur.movsesyan@utt.fr
}

\begin{abstract}
We evaluate experimentally and theoretically the role of the residual ligands and ambient environment refractive index on the optical response of a single spherical gold nanoparticle on a substrate and demonstrate the changes in the near and far-field properties of its hybridized modes in the presence of the CTAB layer. Particularly, we show that the conventional bilayer scheme for CTAB is not relevant for colloidal nanoparticles deposited on a substrate. We show that this CTAB layer considerably changes the amplitude and localization of the confinement of electric field which is of prime importance in the design of plasmonic complex systems coupled to emitters. Moreover, we numerically study the influence of CTAB layer on the modification of sensitivity of plasmonic resonances of a gold nanopshere to local refractive index changes.

(c) 2019 Optical Society of America
\end{abstract}

\section{Introduction}

The optical properties of metallic nanoparticles attract a huge attention of scientific community thanks to so-called localized surface plasmon resonances (LSPR). These resonances are the collective oscillation of free electrons on the metal surface driven by an external electric field. The LSPR excitations result in an increase of light scattering and absorption cross-sections, high electromagnetic field localization in the vicinity of the metallic nanoparticle. The LSPR spectral peak position and its form strongly depend on many factors such as the nanoparticle size, shape, material, as well as on the refractive index of surrounding medium. The LSPR spectral tunability and high electric field enhancement near the metallic nanoparticles open a wide area of applications in bio or chemical sensing, surface enhanced spectroscopies, lasing, local thermal treatment, color generation and etc [1-10].

There is a variety of methods for metallic nanoparticles fabrication providing nanostructures with high or low level of shape and size control [11]. Chemical synthesis of the colloidal nanoparticles is one of the most studied and applied technique due to the high quality of the obtained metal nanocrystals, high control of its size, shape and low-cost [12]. Amongst all other shapes (rods, stars, bipyramids and others) nanospheres are often studied for interparticle coupling [13-16] and enhancement mechanisms [17-19] due to highly developed analytical and numerical calculation methods of its properties as well as advanced wet-chemistry approaches.

Colloidal nanoparticles of the size more than tens of nm are usually grown using seed-mediated growth method [20,21]. In this case the synthesis and stabilization are often performed in cetyltrimethylammonium bromide (CTAB) solution. A fraction of the CTAB molecules is bound to the surface of the nanoparticles in the form of a bilayer while the rest of the molecules are in 
solution and can form micelles when the concentration is greater than $10^{-3} \mathrm{M}$ [22]. After the deposition on the substrate, the CTAB molecules may still surround metal nanoparticles and induce changes in their optical properties, which are not usually taken into account. Firstly, CTAB molecules increase the local refractive index near the plasmonic nanoparticle. Secondly, it may change the effectiveness of the surface enhanced fluorescence or Raman scattering (SERS) due to the additional distance between the nanoparticle and emitter. Also, the CTAB layer changes symmetry of the system of the nanoparticle on the substrate and therefore the field localization and the spectral position of plasmonic modes. It was reported previously that the asymmetry of the environment may cause hybridization of the plasmonic modes of the nanoparticle if their spectral positions are close [23]. This effect was studied for different metallic nanoparticles [23-27], however the possible modulation of the optical properties due to the CTAB layer around the nanoparticles was not considered. Also, important to note that in majority of studies the optical properties of the metallic nanospheres are simulated using Mie theory and effective refractive index approximation which does not include all mentioned effects. In this paper, we used FDTD simulations and apply our collection geometry (experimental numerical aperture) to be as close as possible to the experiments. The FDTD calculations show realistic distribution of the electric field, while for homogeneous effective refractive index the electric field is distributed elsewhere.

In this letter we show the effect of the residual CTAB layer on the near-field and farfield optical properties of chemically synthesized, perfectly spherical nanospheres. These changes can influence on sensing devices based on local refractive indices changes, enhanced spectroscopies (SERS, SEIRA, Surface enhanced fluorescence and etc.) and enhanced photochemistry(photopolymerization, photocatalysis, ..) as they are strongly dependent on near field changes of plasmonic nanoparticles.

\section{Method}

\section{NanoSphere synthesis}

Ultrasmooth and highly uniform Au nanospheres with an average diameter of $180( \pm 2) \mathrm{nm}, 195$ $( \pm 2) \mathrm{nm}$ and $210( \pm 3) \mathrm{nm}$ were synthesized by a seed-mediated growth method following the multi-step procedure reported in Ref. [21]. Single-crystalline Au spheres of increasing sizes were produced through overgrowth of smaller ones. In the first step of growth, a seed solution of 40 $\mathrm{nm}$ spheres was injected in a growth water solution containing $\mathrm{HAuCl}_{4}\left(1.3 \times 10^{-4} M\right)$, ascorbic acid $\left(5.0 \times 10^{-4} M\right)$ and CTAB $\left(1.0 \times 10^{-2} M\right)$. The formed intermediate polyhedrons were treated, using the $\mathrm{Au}(\mathrm{III}) / \mathrm{CTAB}$ oxidant complex, to reshape them into rounded shapes, as explained in details in Ref. [28] and Ref. [21]. Seven to nine successive steps of growth and mild oxidation were required to produce perfectly spherical nanoparticles with an average diameter of $180 \mathrm{~nm}$ to $210 \mathrm{~nm}$ Fig. 1a shows a typical scanning electron micrograph (SEM) of the three solutions of gold nanospheres.

\section{Sample preparation}

In order to locate gold nanospheres on a substrate, we developed a grid mesh of $50 \mu \mathrm{m}$ squares on a borosilicate glass substrate by a UV lithography (shown Fig 1.b) [29]. We spin-coated a positive MICROPOSIT S1813 (MicroChem) photoresist on a glass slide to curve the grid lines and number by mask aligner MJB4 (Suss MicroTec). Therefore, the exposed area (lines and numbering) was removed by MF319 developer. Hence, a silica layer was deposited by electron beam evaporation. The residual photoresist was removed in acetone. Finally, the sample was coated by $70 \mathrm{~nm}$ ITO (indium-tin oxide) layer by a commercial company (Solems S.A.). We characterized the substrate morphology by an atomic force microscope (inset Fig. 1b). Indeed, the calculated roughness is very small $(\sim 1 \mathrm{~nm})$. Then, the substrate was exposed during 10 min to an UV ozone lamp to make the surface hydrophilic. Right after, a droplet $(2 \mu l)$ of a 

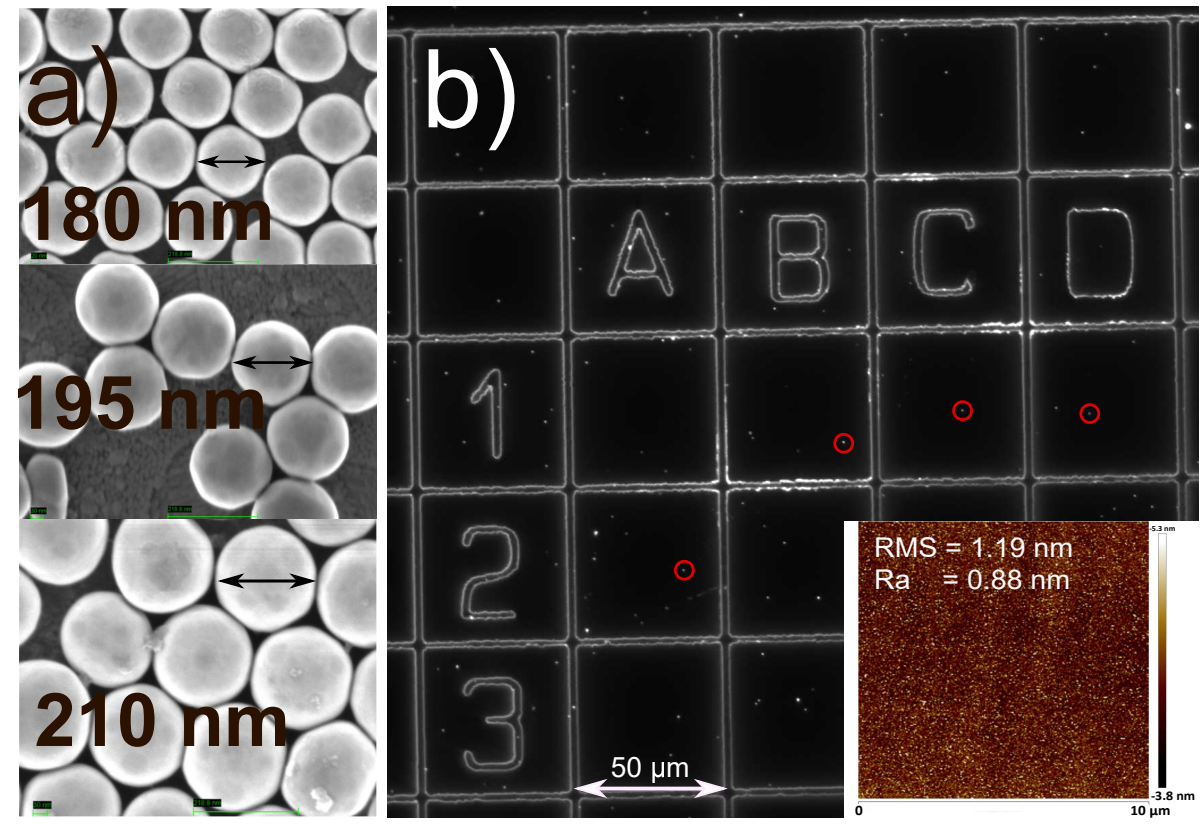

Fig. 1. (a) SEM images of gold nanospheres of different diameters. (b) Dark-field image of gold nanospheres deposited on grid masked ITO substrate. Red circles show examples of presence of nanospheres. Inset shows a characterized ITO substrate by atomic form microscope (AFM) before the deposition of gold nanospheres and the calculated roughness of the substrate (root mean squared -RMS, arithmetic average - Ra).

water-diluted solution of gold nanospheres was deposited and left to dry. The solution was diluted to yield a nanosphere density of 1 sphere $/ 10 \mu \mathrm{m}^{2}$, so no significant nanoparticle field coupling was expected. The substrate was rinsed during about $15 \mathrm{sec}$ with ethanol and dried out (three consecutive times) and then put for 30 min under the UV-ozone lamp in order to remove the CTAB surfactant both from the surface of the nanosphere and from the ITO surface too. After the UV-ozone treatment the substrate was rinsed again in ethanol for $15 \mathrm{sec}$ and dried (three consecutive times). The nanoparticles cleaned using this protocol ("one cycle of cleaning") was firstly studied. In the further experiments to completely remove the CTAB molecules seven repeating cleaning cycles were performed.

\section{Characterization}

We use a dark-field optical microscope Zeiss Axio Imager Z2 for single gold nanospheres characterization in reflection geometry. A dark-field objective of 50x magnification with a numerical aperture 0.8-0.95 (53.1 - 71.8 degrees) illuminates the sample, then the same objective collects the scattered light with a numerical aperture of 0.8 [30]. Therefore, we couple the objective with $50 \mu \mathrm{m}$ core optical fiber in order to filter spatially the collection zone, which will include only the scattering of a single nanoparticle. Hence, the fiber is connected to Ocean Optics QE65000 spectrometer. The final scattering spectrum is obtained using a method described in Ref. [30]. Scattering spectra and near field maps are calculated with the help of the commercial software "FDTD Solution" from Lumerical, based on finite difference time domain method. We use perfect matching layer (PML) as the boundary conditions on the wall sides of the simulation box [31]. The incident light is injected from the air side using the TFSF (total field scattered field) source [32]. The discretization mesh is $2 \mathrm{~nm}$ over the gold nanospheres. In order to obtain 
scattering spectrum for a numerical aperture of 0.8 , we calculate the far-field (k-space) maps for each wavelength at reflection geometry, therefore, we sum the scattered field (intensities) of all angles below 53.1 for each wavelength. For the numerical simulations the refractive index of CTAB layer is considered 1.435 [33].

\section{Results and discussion}

In Fig. 2a and $2 b$ we depict the reflection scattering spectra of GNSs of similar $180 \mathrm{~nm}$-diameters (measured by SEM presented in insets). One may see that the two spectra are different although the SEM images show similar diameters. For the first nanosphere (Fig. 2a) the main peak is relatively narrow and has maximum at $600 \mathrm{~nm}$. For the second nanosphere (Fig. 2b), the main peak is broader with a maximum near $650 \mathrm{~nm}$.

\section{Experiment}
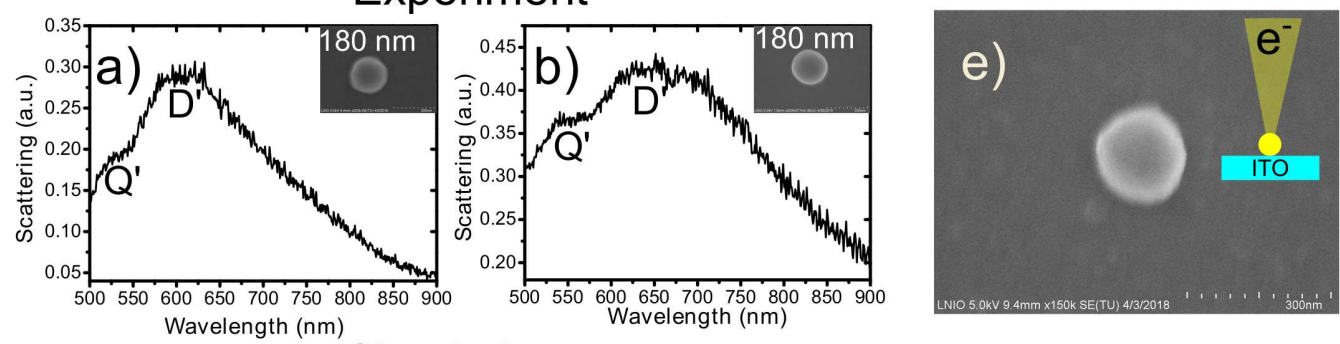

\section{Simulation}
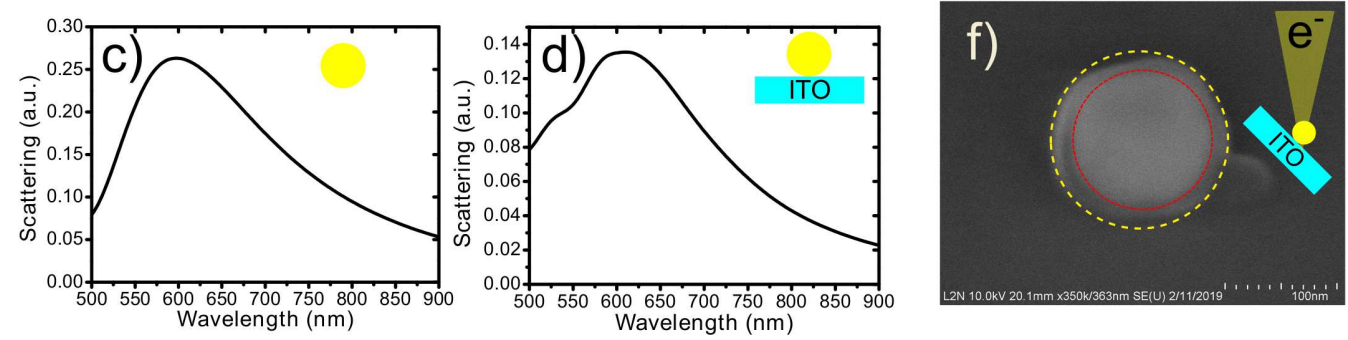

Fig. 2. (a) and (b) scattering spectra of similar gold $180 \mathrm{~nm}$-diameter nanospheres, the collection is in reflection geometry, and the numerical aperture is 0.8. (c) Simulated scattering (reflection geometry) spectrum of GNS of $180 \mathrm{~nm}$-diameter placed in air. (d) Simulated scattering (reflection geometry) spectrum of GNS of $180 \mathrm{~nm}$-diameter deposited on the ITO substrate. For numerical simulations, the numerical aperture is applied on the simulated spectrum (b). SEM image of a gold nanosphere at (e) normal and at 45 degree tilted to the electron beam.

To understand these differences, we perform numerical simulation of reflection scattering spectra of a $180 \mathrm{~nm}$-diameter GNS in air and on an ITO substrate and present them on Fig. 2c and $2 \mathrm{~d}$ respectively. In the calculations we chose a perfect GNS. The calculation of the scattering spectrum for a single nanosphere placed in air shows a broad single peak (Fig 2c). When an ITO substrate is present, the main peak is redshifted to $610 \mathrm{~nm}$ and a shoulder appears at $540 \mathrm{~nm}$ which may reflect the emergence of a new plasmonic mode (Fig. 2d). A similar behavior for the plasmonic nanospheres was reported previously [34].

The presence of a substrate changes the local refractive index near the plasmonic nanoparticles, and then induces a spectral shift of all modes. In the same time, the substrate breaks the symmetry of the system and may cause a coupling between bright dipolar and dark quadrupolar modes if they overlap spectrally [23]. Although not shown here calculations of the scattering spectrum for a gold nanosphere deposited on a glass substrate shows a single peak $(602 \mathrm{~nm})$ like in air case. A 
higher refractive index of ITO ( 1.8) compared to glass ( 1.5) redshifts the plasmonic modes of a sphere further than in the glass case. One may assume that the quadrupolar mode of GNS deposited on glass is damped by strong inter-band transitions (below $500 \mathrm{~nm}$ ) of gold, while the ITO substrate redshifts the quadrupolar mode further away from the interband spectral zone. Then the dipolar and quadrupolar may interact and after hybridization both modes appear in the scattering spectrum (Fig. 2d).

The local refractive index usually has significant impact on the LSPR spectral position but comparing the scattering spectra in Fig. $2 \mathrm{c}$ and Fig. $2 \mathrm{~d}$ one may notice only a minor shift (13 $\mathrm{nm}$ ) of the main mode in the presence of the ITO substrate. The asymmetrical environment causes redistribution of the electric field confinement of plasmonic modes of nanoparticle. The confinement of electric field of nanoparticles deposited on a substrate is usually concentrated near the surface for dipolar mode. Moreover, an electron cloud oscillating in higher refractive index of substrate than superstrate (air) result in a redshift of plasmonic modes due to reduction of polarization charges, therefore the restoring force and the frequency $[35,36]$. This reduction of polarization charges depends on the intersection of the nanoparticle-surface interfaces. Then redshift of the resonance position depends strongly on the area of the contact between the plasmonic particle and the substrate. The GNS has a small touching area considering also the high quality of ITO substrate (averaged roughness $\sim 1 \mathrm{~nm}$ ), accordingly we do not see a significant redshift (from $597 \mathrm{~nm}$ to $610 \mathrm{~nm}$ ) of the dipolar mode peak position although a small spectral shift was enough to bring out the quadrupolar mode away from strong inter-band transition region.

We observe the separation of the two modes on the experimental scattering spectra (Fig.2a-b). The modes are denoted as D' and Q'. One may notice a good agreement between simulation and experiment comparing Fig. 2a and 2d. However, Fig. $2 b$ differs in shape and position of the peak from the calculated one (Fig 2d). To understand the difference between the calculated and experimental spectra we performed SEM study for 45-degree tilted sample. In Fig. 2e the image of nanosphere taken at normal angle is presented (the diameter is $150 \mathrm{~nm}$ ), while the tilted measurement (Fig. 2f) shows a sphere with an Au core of $125 \mathrm{~nm}$ and a shell of around 10-12 nm. It is important to note that after the fabrication of the gold nanospheres and their deposition on the substrate, the samples are usually washed from CTAB using "one cycle of cleaning". However, some residue of CTAB may still be attached to the nanosphere, which we see on the SEM images, and influence on the optical response of nanospheres. Generally, thickness of CTAB layer adsorbed at the surface of nanoparticles is measured approximately 3 $\mathrm{nm}$ in a solution [37], but when we deposit them on the substrate due to surface tensions and capillary forces many layers may coat the nanoparticles. Therefore, the thickness of the CTAB is much larger for dried sample than in a solution.

In order to investigate the influence of CTAB on the optical response of GNS, we recorded their scattering spectra after one and seven cycles of cleaning from CTAB. The SEM images are taken after all optical measurements i.e. after the seven cycles of cleaning, as the optical properties of GNS can be changed due to the contamination caused by electron beam exposure, which has been already reported $[38,39]$. Fig. 3 shows the experimental and simulated scattering spectra for GNS of three diameters $(180,195,210 \mathrm{~nm})$. In Fig. 3a we observe that the scattering spectrum of $180 \mathrm{~nm}$ diameter GNS highlights two well-defined plasmonic bands denoted as D and Q. After repetitive washing from CTAB the spectrum of the same GNS shows two plasmonic bands (Q' and D') blue-shifted relatively to Fig. 3a. Indeed, CTAB having a higher refractive index (1.435) than air changes the surrounding effective refractive index, which results in the red-shifting of plasmonic modes. In order to claim that it is due to complete removal of CTAB, we did the scattering simulations for a GNS deposited on the ITO substrate without residual CTAB and coated with $8 \mathrm{~nm}$ of CTAB depicted respectively on Fig. 3d and 3c.

The good match between the simulated and experimentally obtained scattering spectra supports 


\section{Experiment}
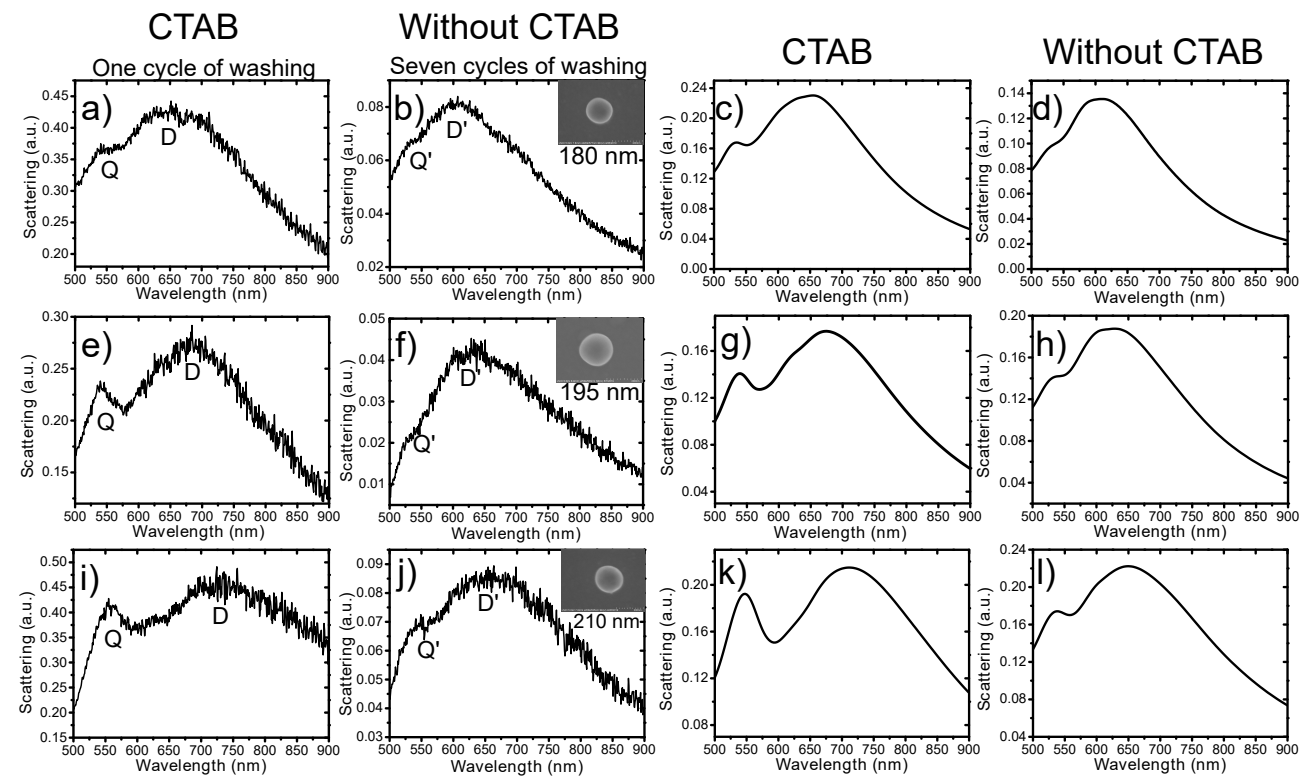

Fig. 3. Experimental scattering spectra of gold nanospheres of different diameters (180 $\mathrm{nm}, 195 \mathrm{~nm}$ and $205 \mathrm{~nm}$ ) with residual CTAB layer after one cycle of cleaning (a, e, i) and after complete CTAB removal due to seven cycles of cleaning $(b, f, j)$ and corresponding simulated spectra (c, b, g, h, k, l).

our explanation. For precise calculations of the scattering spectra, we used the actual collection angle (53 degrees-half angle) of the objective in the simulations. We also did analogous studies for other sizes of GNS which are presented in Fig. 3e, f, g, h for $195 \mathrm{~nm}$-diameter GNS and in Fig. 3i, j, k, 1 for $210 \mathrm{~nm}$-diameter respectively. Herein we also observe a good match between experimental and simulated spectra of GNS before and after the additional washing from CTAB.

While the far-field properties are important for the determination of LSPR of metallic nanostructures, it is as well as important to be aware of the near-field changes induced by the CTAB layer. With the help of near-field FDTD simulations we draw the near-field maps of 180 nm-diameter GNS without and with CTAB layer on Fig. 4 a-d for two plasmon bands attributed to quadrupolar and dipolar modes.

The asymmetry of the system environment brings in the electromagnetic field concentration at the intermedium of the nanoparticle and the substrate which has higher refractive index than air. These modes do not show the usual character of dipolar and quadrupolar modes as they are hybridized modes. Indeed, they do not have the well-known two and four lobes, respectively.

The near-field confinement and magnitude at the vicinity of the nanoparticle is crucial for chemical-bio sensing. When we compare near- field maps of nanoparticles without (Fig. 4a,4b) and with the CTAB layer (Fig. 4c,4d), we clearly see that the maximum of local electric field of mode Q' and Mode D' near the GNS without dielectric layer prevails over the field of mode Q and Mode D near the GNS with a CTAB layer. For global sensing or enhanced spectroscopies, the species are not placed only at the maxima of the electric field but deposited all around the nanoparticle. Then, we calculated the averaged field intensity in a zone of $20 \mathrm{~nm}$ thick near the nanoparticle surface and we present it on Fig. $4 \mathrm{e}$ and $4 \mathrm{f}$.

Note, that for the GNS with CTAB layer, the averaged intensity is calculated with values beyond the CTAB layer. The analysis of the averaged near field intensity of two plasmon bands 

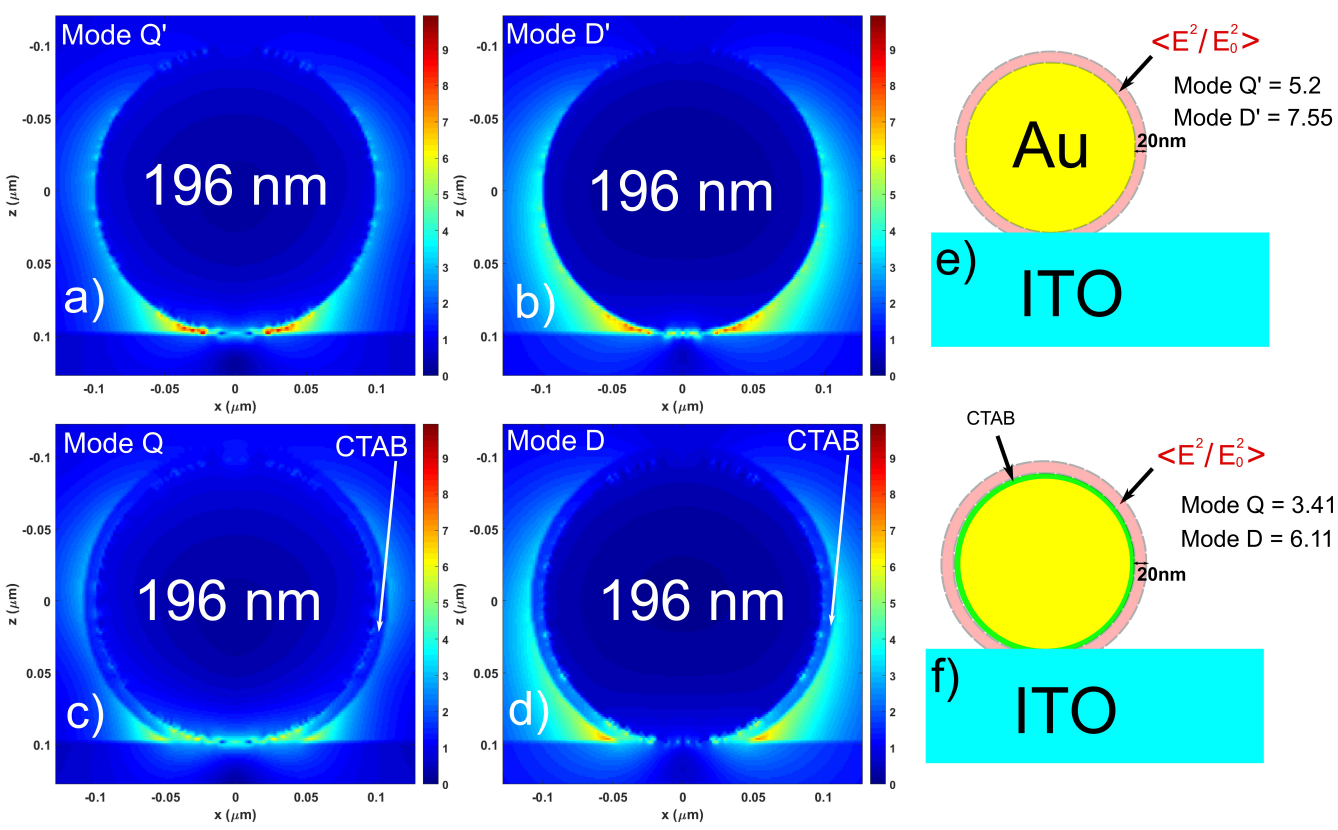

Fig. 4. Near-field maps of $196 \mathrm{~nm}$-diameter gold nanosphere deposited on a ITO substrate for (a) Mode Q' and (b) Mode D' in absence of residual CTAB compared to covered with CTAB $10 \mathrm{~nm}$ layer for (c) Mode Q and (d) Mode D. The intensities of the maps are normalized at the same scale. Calculated averaged intensities of electric field in a zone (colored pink) of $20 \mathrm{~nm}$ next to the particle for (e) mode D' and Q', (f) mode D and Q.

for GNS with or without CTAB shows that the near field of both modes are larger for GNS without the residual CTAB.

The intensity of the electric field confined around a metallic nanoparticle is a key parameter for enhanced spectroscopies and photocatalytic processes, therefore the decrease of the electric field seeks to the dropping of efficiencies of these applications. One of well-studied LSPR applications are bio-chemical sensors based on LSPR changes depending on refractive index modification of surrounding medium [6,40-44]. In order to understand how the CTAB layer influence on these applications, we do numerical study furthermore.

In Fig. $5 \mathrm{a}$ and $5 \mathrm{~b}$ we show scattering spectra of $125 \mathrm{~nm}$-diameter GNS without and with CTAB layer deposited on ITO substrate and embedded in media with refractive index "n" respectively. We consider the constant refractive index for a CTAB layer with a thickness more than $8 \mathrm{~nm}$ [33]. The insets show the schematics of two systems. We chose the size of nanosphere corresponding to the recorded by SEM in Fig. 2e and 2f, in order to not have very wide resonances. Moreover, the small size GNS exhibits only dipolar mode on scattering spectra, which makes easier to determine the peak positions and the widths of them. We vary the refractive index of media (superstrate) from 1.33 to 1.4 in order to define sensitivity of both systems (with and without CTAB). Visually, one may observe that the GNS without CTAB shows better sensitivity than with CTAB (Fig. 5b).

Fig. 5c shows the dependence of LSPR peak position on surrounding refractive index (n). From these curves we can easily determine the sensitivity of these two systems, which are $213 \mathrm{~nm}$ $R I U^{-1}$ without CTAB and $122 \mathrm{~nm} R I U^{-1}$ with CTAB. The sensitivity is calculated by following formulation: $S=\Delta \lambda / \Delta n$, where $\Delta \lambda$ is peak position shift caused by $\Delta n$ refractive index shift [43]. We claim that a CTAB layer worsen the sensitivity about 1.7 folds. We also looked another parameter that define the sensitivity of the system called figure of merit (FOM), calculated by the 

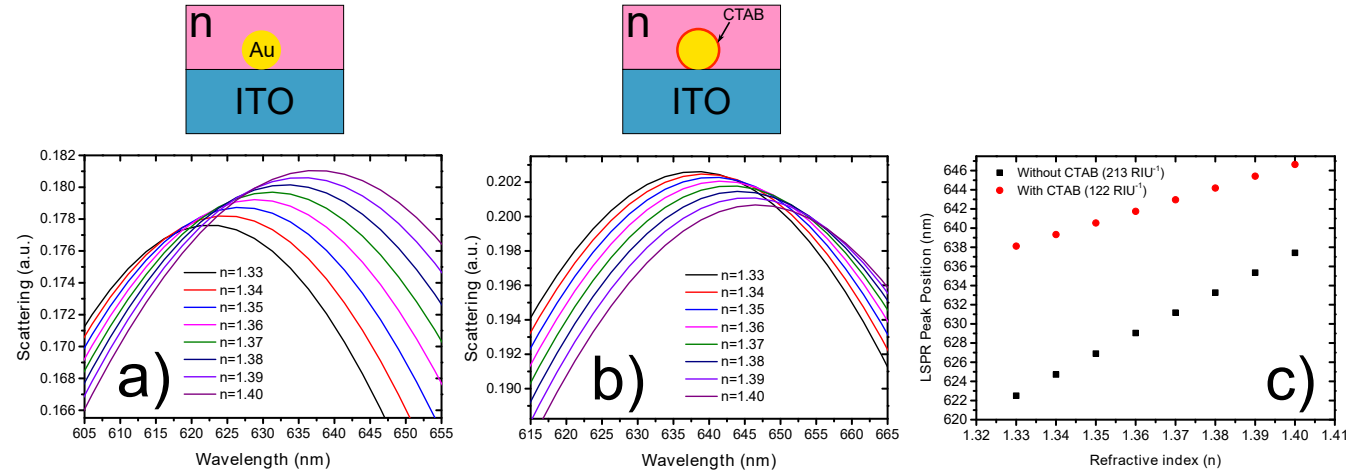

Fig. 5. (a) Calculated scattering spectra of gold $125 \mathrm{~nm}$-diameter nanosphere embedded in medium wiht refractive index $\mathrm{n}$.(b) Calculated scattering spectra of gold $125 \mathrm{~nm}$-diameter nanosphere covered with $12 \mathrm{~nm}$ CTAB layer and embedded in medium with refractive index n. (c) Dependence of LSPR peak position on refractive index for cases (a) and (b).

ratio between sensitivity and the full width at half maximum of LSPR. The GNSs without CTAB shows FOM from 0.8-1.1, while the GNSs with CTAB have FOM from 0.41-0.43 for a range of refractive index of 1.33-1.4. One may declare that FOM is diminished about 2 times due to CTAB layer. The GNS with a CTAB layer sustains wider widths of resonances due to higher effective refractive index as these resonances are redshifted compared with the system without CTAB. Therefore, FOM for GNS with CTAB layer is smaller.

The sensitivity shows the rate of the changes of $\Delta \lambda$ due to changes of superstrate refractive index $\Delta n$. Similarly the changes of effective refractive index $\Delta n_{\text {eff }}$ is proportional to $\Delta \lambda[45,46]$. Although, the effective refractive index for GNS with CTAB is higher than without, the $\Delta n_{\text {eff }}$ for GNS with CTAB is smaller than for GNS without CTAB since the sensitivity and $\Delta \lambda$ is smaller.

\section{Conclusion}

We successfully demonstrated the importance of the residual ligands that are used for synthesis of metallic nanoparticles on the far-field and near-field optical responses. We think that these effects, which generally have not been considered previously, should be taken into account for future studies. We showed, that the standard bilayer scheme for CTAB molecules on metallic nanoparticles is not relevant, when they are deposited on the substrate. Furthermore, the residual ligands damp the near-field average amplitude and modify the electric field localization around the nanoparticle. This modification of the near-field can directly influence on enhanced fluorescence and enhanced vibrational spectroscopies as they are strongly dependent on the near-field of plasmonic nanoparticles. Also, for enhanced Raman spectroscopy, the residual ligands may exhibit non-expected Raman lines besides the Raman lines of species under investigation.

In the case of the numerous studies dedicated to the characterization of the photoinjection of hot charge carriers (electron or holes) within gold-nanohybrids, the presence or not of a residual layer of $\mathrm{CTAB}$ at the interface is a key parameter that is generally not taken into account, although of crucial importance for this photoinduced process. We also show importance of cleaning of residual CTAB layer in order to improve the sensitivity of resonances to local refractive index changes, which is crucial in bio-chemical sensing.

\section{Acknowledgment}

The authors acknowledge th Nano'Mat platform for nanofabrication facilities. We express our gratitude to Régis Déturche for AFM measurements. The The numerical simulations were 
supported by the HPC Center of Champagne-Ardenne ROMEO. We acknowledge gratefully "Ministère de l'Education Nationale, de l'Enseignement Supérieur et de la Recherche" for providing the Doctoral fellowship.

\section{Disclosures}

The authors declare that there are no conflicts of interest related to this article.

\section{References}

1. S. A. Maier, Plasmonics: fundamentals and applications (Springer, New York, 2007).

2. L. Novotny and B. Hecht, Principles of nano-optics (Cambridge University Press, Cambridge, 2009).

3. E. A. Coronado, E. R. Encina, and F. D. Stefani, "Optical properties of metallic nanoparticles: manipulating light, heat and forces at the nanoscale," Nanoscale 3, 4042-4059 (2011).

4. S. Vaschenko, A. Ramanenka, O. Kulakovich, A. Muravitskaya, D. Guzatov, A. Lunevich, Y. Glukhov, and S. Gaponenko, "Enhancement of labeled alpha-fetoprotein antibodies and antigen-antibody complexes fluorescence with silver nanocolloids," Procedia Eng. 140, 57-66 (2016).

5. D. Kim, "Effect of resonant localized plasmon coupling on the sensitivity enhancement of nanowire-based surface plasmon resonance biosensors," J. Opt. Soc. Am. A 23, 2307 (2006).

6. S. J. Zalyubovskiy, M. Bogdanova, A. Deinega, Y. Lozovik, A. D. Pris, K. H. An, W. P. Hall, and R. A. Potyrailo, "Theoretical limit of localized surface plasmon resonance sensitivity to local refractive index change and its comparison to conventional surface plasmon resonance sensor," J. Opt. Soc. Am. A 29, 994 (2012).

7. L. Cognet, M. Treguer-Delapierre, and S. Link, "Biological applications of electromagnetically active nanoparticles," J. Phys. D: Appl. Phys. 50, 200201 (2017).

8. P. Winkler, M. Belitsch, A. Tischler, V. Häfele, H. Ditlbacher, J. R. Krenn, A. Hohenau, M. Nguyen, N. Félidj, and C. Mangeney, "Nanoplasmonic heating and sensing to reveal the dynamics of thermoresponsive polymer brushes," Appl. Phys. Lett. 107 (2015).

9. N. Zhou, V. López-Puente, Q. Wang, L. Polavarapu, I. Pastoriza-Santos, and Q. H. Xu, "Plasmon-enhanced light harvesting: Applications in enhanced photocatalysis, photodynamic therapy and photovoltaics," RSC Adv. 5, 29076-29097 (2015).

10. W. Zhou, M. Dridi, J. Y. Suh, C. H. Kim, D. T. Co, M. R. Wasielewski, G. C. Schatz, and T. W. Odom, "Lasing action in strongly coupled plasmonic nanocavity arrays," Nat. Nanotechnol. 8, 506-511 (2013).

11. N. C. Lindquist, P. Nagpal, K. M. McPeak, D. J. Norris, and S.-H. Oh, "Engineering metallic nanostructures for plasmonics and nanophotonics," Reports on Prog. Phys. 75, 36501 (2012).

12. L. Shao, Y. Tao, Q. Ruan, J. Wang, and H. Q. Lin, "Comparison of the plasmonic performances between lithographically fabricated and chemically grown gold nanorods," Phys. Chem. Chem. Phys. 17, 10861-10870 (2015).

13. S. K. Ghosh and T. Pal, "Interparticle coupling effect on the surface plasmon resonance of gold nanoparticles: From theory to applications," Chem. Rev. 107, 4797-4862 (2007).

14. S. J. Barrow, X. Wei, J. S. Baldauf, A. M. Funston, and P. Mulvaney, “The surface plasmon modes of self-assembled gold nanocrystals," Nat. Commun. 3, 1275-1279 (2012).

15. L. Chuntonov and G. Haran, "Trimeric plasmonic molecules: the role of symmetry," Nano Lett. 11, 2440-2445 (2011).

16. P. Nordlander, C. Oubre, E. Prodan, K. Li, and M. I. Stockman, "Plasmon hybridization in nanoparticle dimers," Nano Lett. 4, 899-903 (2004).

17. V. V. Klimov and D. V. Guzatov, "Strongly localized plasmon oscillations in a cluster of two metallic nanospheres and their influence on spontaneous emission of an atom," Phys. Rev. B - Condens. Matter Mater. Phys. 75, 1-7 (2007).

18. D. V. Guzatov, S. V. Vaschenko, V. V. Stankevich, A. Y. Lunevich, Y. F. Glukhov, and S. V. Gaponenko, "Plasmonic enhancement of molecular fluorescence near silver nanoparticles : theory, modeling, and experiment," J. Phys. Chem. C 116, 10723-10733 (2012).

19. K. Q. Le, "Nanoplasmonic enhancement of molecular fluorescence: theory and numerical Modeling," Plasmonics 10, 475-482 (2015).

20. N. R. Jana, L. Gearheart, and C. J. Murphy, "Seeding growth for size control of 5-40 nm diameter gold nanoparticles," Langmuir 17, 6782-6786 (2001).

21. Q. Ruan, L. Shao, Y. Shu, J. Wang, and H. Wu, "Growth of monodisperse gold nanospheres with diameters from 20 $\mathrm{nm}$ to $220 \mathrm{~nm}$ and their core/satellite nanostructures," Adv. Opt. Mater. 2, 65-73 (2014).

22. S. Lee, L. J. Anderson, C. M. Payne, and J. H. Hafner, "Structural transition in the surfactant layer that surrounds gold nanorods as observed by analytical surface-enhanced raman spectroscopy," Langmuir 27, 14748-14756 (2011).

23. S. Zhang, K. Bao, N. J. Halas, H. Xu, and P. Nordlander, "Substrate-induced Fano resonances of a plasmonic nanocube: a route to increased-sensitivity localized surface plasmon resonance sensors revealed," Nano Lett. 11, $1657-1663$ (2011).

24. Y. Wu and P. Nordlander, "Finite-difference time-domain modeling of the optical properties of nanoparticles near," J. Phys. Chem. C 114, 7302-7307 (2010). 
25. H. Chen, L. Shao, T. Ming, K. C. Woo, Y. C. Man, J. Wang, and H. Q. Lin, "Observation of the Fano resonance in gold nanorods supported on high-dielectric-constant substrates," ACS Nano 5, 6754-6763 (2011).

26. F. Qin, X. Cui, Q. Ruan, Y. Lai, J. Wang, H. Ma, and H. Q. Lin, "Role of shape in substrate-induced plasmonic shift and mode uncovering on gold nanocrystals," Nanoscale 8, 17645-17657 (2016).

27. M. W. Knight, Y. Wu, J. B. Lassiter, P. Nordlander, and N. J. Halas, "Substrates matter: influence of an adjacent dielectric on an individual plasmonic nanoparticle," Nano Lett. 9, 2188-2192 (2009).

28. J. Rodríguez-Fernández, J. Pérez-Juste, P. Mulvaney, and L. M. Liz-Marzán, "Spatially-directed oxidation of gold nanoparticles by Au(III)-CTAB complexes," J. Phys. Chem. B 109, 14257-14261 (2005).

29. W. Chaâbani, J. Proust, A. Movsesyan, J. Béal, A. L. Baudrion, P. M. Adam, A. Chehaidar, and J. Plain, "Large-scale and low-cost fabrication of silicon Mie resonators," ACS Nano 13, 4199-4208 (2019).

30. A. Movsesyan, A.-L. Baudrion, and P.-M. Adam, "Extinction measurements of metallic nanoparticles arrays as a way to explore the single nanoparticle plasmon resonances." Opt. Express 26, 6439-6445 (2018).

31. S. D. Gedney and B. Zhao, "An auxiliary differential equation formulation for the complex-frequency shifted PML," IEEE Transactions on Antennas Propag. 58, 838-847 (2010).

32. K. Abdijalilov and J. B. Schneider, "Analytic field propagation TFSF boundary for FDTD problems involving planar interfaces: Lossy material and evanescent fields," IEEE Antennas Wirel. Propag. Lett. 5, 454-458 (2006).

33. P. Kékicheff and O. Spalla, "Refractive index of thin aqueous films confined between two hydrophobic surfaces," Langmuir 10, 1584-1591 (1994).

34. M. W. Knight, J. Fan, F. Capasso, and N. J. Halas, "Influence of excitation and collection geometry on the dark field spectra of individual plasmonic nanostructures," Opt. Express 18, 2579-2587 (2010).

35. J. P. Kottmann, O. J. Martin, D. R. Smith, and S. Schultz, "Field polarization and polarization charge distributions in plasmon resonant nanoparticles," New J. Phys. 2 (2000).

36. H. Wang, "Plasmonic refractive index sensing using strongly coupled metal nanoantennas: Nonlocal limitations," Sci. Reports 8, 1-8 (2018).

37. S. Gómez-Graña, F. Hubert, F. Testard, A. Guerrero-Martínez, I. Grillo, L. M. Liz-Marzán, and O. Spalla, "Surfactant (Bi) layers on gold nanorods," Langmuir 28, 1453-1459 (2012).

38. S. Hettler, M. Dries, P. Hermann, M. Obermair, D. Gerthsen, and M. Malac, "Carbon contamination in scanning transmission electron microscopy and its impact on phase-plate applications," Micron 96, 38-47 (2017).

39. A. J. Griffiths and T. Walther, "Quantification of carbon contamination under electron beam irradiation in a scanning transmission electron microscope and its suppression by plasma cleaning," J. Physics: Conf. Ser. 241, 0-4 (2010).

40. S. Szunerits and R. Boukherroub, "Sensing using localised surface plasmon resonance sensors," (2012).

41. N. A. Mortensen, S. Xiao, and J. Pedersen, "Liquid-infiltrated photonic crystals: Enhanced light-matter interactions for lab-on-a-chip applications," Microfluid. Nanofluidics 4, 117-127 (2008).

42. K. M. Mayer and J. H. Hafner, "Surface plasmon resonance sensors: review," Sensors Actuators B: Chem. 54, 3-15 (1999).

43. K. M. Mayer and J. H. Hafner, "Localized surface plasmon resonance sensors," Chem Rev 111, 3828-3857 (2011).

44. C. Yu and J. Irudayaraj, "Quantitative evaluation of sensitivity and selectivity of multiplex nanoSPR biosensor assays," Biophys. J. 93, 3684-3692 (2007).

45. P. Kvasnička and J. Homola, "Optical sensors based on spectroscopy of localized surface plasmons on metallic nanoparticles: Sensitivity considerations," Biointerphases 3, FD4-FD11 (2008).

46. G. Barbillon, J.-L. Bijeon, J. Plain, M. Lamy De La Chapelle, P.-M. Adam, and P. Royer, "Biological and chemical gold nanosensors based on localized surface plasmon resonance," Gold Bull. 40, 240-244 (2007). 